\title{
Avaliação do nível de estresse em cadelas de abrigo submetidas a um período de adaptação de sete dias em canis experimentais
}

\author{
[Evaluation of the stress level in female shelter dogs undergoing a seven-day adaptation period in \\ experimental kennels]
}

\section{"Artigo Científico/Scientific Article"}

\section{Fernanda Vieira Henrique $^{1 *}$, Roberta Nunes Parentoni ${ }^{2}$, Angélica Ramalho Araújo Leite ${ }^{3}$, Dayvid Vianês Farias Lucena ${ }^{4}$, Rodrigo Gustavo Dantas dos Santos ${ }^{3}$, Almir Pereira de Souza ${ }^{5}$, Pedro Isidro da Nóbrega Neto}

\author{
${ }^{1}$ Programa de Residência Multiprofissional, Universidade Federal de Campina Grande (UFCG), Patos-PB, Brasil. \\ ${ }^{2}$ Universidade Federal da Paraíba (UFPB), João Pessoa-PB, Brasil. \\ ${ }^{3}$ Médico(a) veterinário(a) autônomo(a). \\ ${ }^{4}$ Universidade Estadual Paulista (UNESP), Campus de Jaboticabal-SP, Brasil. \\ ${ }^{5}$ Unidade Acadêmica de Medicina Veterinária, Universidade Federal de Campina Grande (UFCG), Patos-PB, Brasil. \\ *Autor para correspondência/Corresponding author: E-mail: nandinhavh@gmail.com
}

\section{Resumo}

Objetivou-se avaliar o nível de estresse em cadelas em canis experimentais durante sete dias. Foram utilizadas

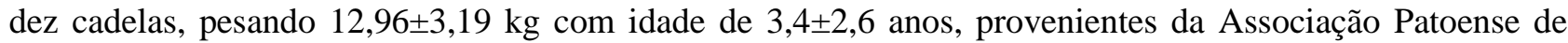
Proteção aos Animais. Amostras de sangue foram coletadas para determinação do cortisol e da glicemia. Foram mensuradas, frequências cardíaca e respiratória, temperatura retal e pressões arteriais sistólica, média e diastólica. Para avaliar clinicamente o estresse foi utilizado um Escore Composto de Estresse. O estresse obtido foi classificado em discreto (escores de 0 a 6), moderado (escores de 7 a 14) e intenso (escores >14). Na análise estatística utilizou-se o programa computacional Bioestat 5.0 ao nível de 5\% de significância. Observou-se um decréscimo do cortisol, porém, estatisticamente, apenas $144(0,60 \pm 0,67 \mu \mathrm{g} / \mathrm{dL})$ e $168 \mathrm{horas}$ $(0,55 \pm 0,55 \mu \mathrm{g} / \mathrm{dL})$ após a chegada dos animais ao canil o cortisol diferiu do momento basal $(2,78 \pm 2,7 \mu \mathrm{g} / \mathrm{dL})$. $\mathrm{O}$ estresse foi classificado em discreto em todos os momentos (escores <6). As cadelas deste experimento não perceberam a admissão ao canil como um potencial estressor. Sugere-se que seis dias são suficientes para cadelas se adaptarem a um novo ambiente.

Palavras-chave: canino; cortisol; glicemia.

\begin{abstract}
The objective of this study was to evaluate the stress level in female dogs in experimental kennels for seven days. Ten female dogs, weighing $12.96 \pm 3.19 \mathrm{~kg}$ and aged 3.4 \pm 2.6 years, from the Patoense Association for the Protection of Animals were used. Blood samples were collected to determine cortisol and blood glucose levels. Heart and respiratory rate, rectal temperature, and systolic, mean and diastolic arterial pressure were measured. To clinically evaluate stress, we used a Stress Composite Score. Stress was classified as discreet (score 0 to 6), moderate (scores 7-14), and intense (score >14). Statistical analyses were performed using the computer program Bioestat 5.0 at a 5\% significance level. There was a decrease in the cortisol; however, statistically, only $144(0.60 \pm 0.67 \mu \mathrm{g} / \mathrm{dL})$ and 168 hours $(0.55 \pm 0.55 \mu \mathrm{g} / \mathrm{dL})$ after the arrival of the animals at the kennel did the cortisol differ from the baseline $(2.78 \pm 2.7 \mu \mathrm{g} / \mathrm{dL})$. Stress was classified as discreet in all time points (scores <6). The female dogs of this experiment did not perceive the admission to the kennel as a potential stressor. It is suggested that six days is enough for female dogs to adapt to a new environment.
\end{abstract}

Keywords: canine; cortisol; blood glucose. 


\section{Introdução}

Entre as maiores preocupações relacionadas ao bem-estar em pequenos animais está o estresse imposto ao confiná-los em abrigos ou submetê-los a ambientes diferentes. Em canis, geralmente o cão está confinado em área limitada, sendo obrigado a urinar e defecar naquele espaço. Além disso, sons e odores associados a estresse em outros cães podem contribuir para o desenvolvimento do estresse no novo ambiente. Esse efeito ocorre igualmente em machos, fêmeas, filhotes e animais velhos (Hennessy, 2013), no entanto, uma considerável variação na resposta comportamental ao estresse foi encontrada em cães da mesma raça, idade e sexo, sendo assim infuenciada pela experiência individual do animal (Rooney et al., 2007).

A avaliação do estresse em animais não é fácil e alguns métodos de análise são bastante subjetivos. A atividade do eixo hipotálamopituitária-adrenal (HPA) aumenta em resposta a uma ampla variedade de situações de ameaça ou eventos aversivos, e não pode ser estimada a partir do comportamento. Assim, a mensuração do cortisol pode ser uma variável importante para se avaliar o estado psicológico e o bem-estar de animais, sendo amplamente utilizada para esse propósito (Hennessy et al., 2001; Mormède et al., 2007; Yamamoto et al., 2012; Part et al., 2014). Relata-se que elevações nos níveis de cortisol são observadas nos três primeiros dias quando os animais são levados para abrigos, havendo um declínio gradativo nas concentrações a partir daí (Hennessy et al., 1997). Entre as alterações fisiológicas observadas em situações de estresse destacam-se: aumento na frequência e força de contração cardíacas; na pressão arterial sanguínea (Mcewen e Gianaros, 2010); na frequência respiratória; e na glicemia (Romero e Butler, 2007). A ansiedade e o estresse podem estar relacionados ainda ao aumento da temperatura retal (Yamamoto et al., 2012), como observado por Budziak et al. (2016) em animais de abrigo agitados ou assustados.

Em relação a comportamentos sugestivos de estresse em cães, a literatura cita: aumento da atividade locomotora, apatia, coprofagia, vocalização, poliúria, tremores, lambedura frequente do focinho e comportamentos repetidos ou estereotipados (Beerda et al., 1999; Haverbeke et al., 2008).

A maioria das pesquisas envolvendo animais domésticos, como cães e gatos, têm utilizado animais provenientes de abrigos para animais abandonados, uma vez que os tutores têm cada vez mais não autorizado a participação de seus pets em experimentos. Nessas pesquisas, para que não haja interferência nos resultados experimentais, adotase um período de adaptação, o qual não está totalmente elucidado do ponto de vista temporal, pincipalmente no que diz respeito a animais errantes. Assim, objetivou-se, com este estudo, avaliar o nível de estresse em cadelas de abrigo alojadas em canis experimentais durante sete dias, e determinar se esse período é suficiente para que estas se adaptem a um novo ambiente.

\section{Material e Métodos}

Foram utilizadas dez cadelas adultas, hígidas, pesando 12,96 $\pm 3,19 \mathrm{~kg}$ (média \pm desvio padrão), com idade de $3,4 \pm 2,6$ anos (média \pm desvio padrão), encaminhadas para realização de ováriohisterectomia, provenientes da Associação Patoense de Proteção aos Animais (APPA), localizada na cidade de Patos, Paraíba, Brasil. A higidez dos animais foi determinada com base na avaliação clínica e em exames laboratoriais (hemograma, glicemia de jejum e dosagens de Alanina Aminotransferase - ALT, Aspartato Aminotransferase - AST, ureia e creatinina).

Vale salientar que na APPA os cães, de ambos os sexos e de idades variadas, vivem de forma conjunta em um galpão, onde competem por alimento e espaço, e convivem com animais doentes.

Os animais foram observados no ambiente em que viviam onde foram registrados comportamentos indicativos de estresse, tais como, agressividade, agitação, hiperatividade e apatia. Foram selecionados animais clinicamente saudáveis e que já estavam na APPA há, no mínimo, 15 dias. Os animais foram deslocados da APPA até o Hospital Veterinário da Universidade Federal de Campina Grande, Campus de Patos, às 06:00h da manhã, em caixas de transporte apropriadas para o tamanho do animal e em caminhão coberto em distância de, aproximadamente, cinco quilômetros. Os mesmos ficaram alojados em canis individuais por um período de sete dias, recebendo ração comercial duas vezes ao dia e água ad libitum. Ao chegar ao canil e no último momento de avaliação os animais foram pesados para avaliar se houve ganho ou perda de peso. 
Para mensuração do cortisol, amostras de sangue $(2 \mathrm{~mL})$ foram coletadas por punção da veia jugular, acondicionadas em tubos de ensaio esterilizados sem anticoagulante e centrifugadas por cinco minutos a 5000 rotações por minuto (rpm). A coleta e contenção foram feitas de forma tranquila em todos os animais em um período médio de três minutos. O soro resultante foi armazenado sob refrigeração e, posteriormente, encaminhado ao laboratório para determinação dos valores séricos em $\mu \mathrm{g} / \mathrm{dL}$. A concentração sérica de cortisol foi mensurada pelo método de eletroquimioluminescência (Cassu et al., 2011).

A glicemia em $\mathrm{mg} / \mathrm{dL}$ foi mensurada por meio de glicosímetro portátil (Breeze 2, Bayer HealthCare, Leverkusen, Alemanha) utilizando-se uma gota de sangue colhida para mensuração do cortisol. As coletas de sangue foram realizadas no ambiente em que os animais viviam (M0-momento basal), e a cada 24 horas a partir da chegada dos animais ao canil, totalizando sete dias de avaliação (M24, M48, M72, M96, M120, M144 e M168), entre oito e nove horas da manhã, no primeiro contato com os animais. A aferição dos parâmetros fisiológicos e coleta de sangue basais foram realizadas individualmente, sendo os animais separados em uma sala do abrigo, minimizando-se ao máximo o estresse durante a contenção física. A seleção e separação das cadelas para avaliação durou em média 20 minutos.

Posteriormente à coleta de sangue, quando os momentos coincidiam, os seguintes parâmetros fisiológicos foram mensurados: frequência cardíaca (FC) em batimentos por minuto (bpm), mensurada através de estetoscópio clínico (Estetoscópio clínico, Becton Dickinson Brasil Ltda., São Paulo, SP, Brasil); frequência respiratória $(f)$, mensurada contando-se os movimentos torácicos durante um minuto (mpm); temperatura retal (TR) aferida com apoio de um termômetro clínico digital; e pressões arteriais sistólica (PAS) e diastólica (PAD), mensuradas por método oscilométrico, não invasivo (Medidor de PANI portátil - DL1100, Deltalife, São José dos Campos, SP, Brasil) em que o manguito pneumático foi colocado ao redor do rádio esquerdo, sendo que sua largura correspondia a $40 \%$ da circunferência deste. A cada momento experimental foram realizadas cinco mensurações da pressão arterial, eliminando-se a maior e a menor e, posteriormente, obteve-se a média dos valores restantes, a qual foi anotada como o valor para aquele momento. A pressão arterial média (PAM) foi calculada a partir da fórmula PAM = (PAS - PAD) $/ 3$ + PAD (Massone, 2011). As avaliações paramétricas foram realizadas a cada 12 horas a partir da chegada dos animais ao canil (M12, M24, M36, M48, M60, M72, M84, M96, M108, M120, M132, M144, M156 e M168).

Por último, após descanso de uma hora da aferição dos parâmetros fisiológicos, avaliou-se clinicamente o estresse utilizando-se um Escore Composto de Estresse (ECE) (Tabela 1), escala desenvolvida a partir de comportamentos sugestivos de estresse em cães descritos na literatura (Rooney et al., 2007; Yamamoto et al., 2012; Budziak et al., 2016). Os animais foram avaliados dentro de seus respectivos canis, de forma individual. $\mathrm{O}$ escore máximo obtido com o ECE é de 18 pontos. O estresse obtido por esta escala foi classificado em discreto (escores de 0 a 6), moderado (escores de 7 a 14) e intenso (escores >14). Esta avaliação foi realizada 13 horas (M1) após a chegada dos animais ao canil, e repetida a cada 12 horas, totalizando 14 momentos. Além disso, foi observada a ocorrência de alterações comportamentais e funcionais tais como, modificação na movimentação e/ou no apetite, automutilação, apatia, hiperatividade, tremores, vômito, diarreia, taquipneia, midríase, lambedura frequente dos lábios e nariz e deglutição repetida de saliva, entre outras alterações. As avaliações foram realizadas por um mesmo indivíduo que tinha contato com esses animais apenas nesse momento. Ao final do experimento os animais foram submetidos à cirurgia de ováriohisterectomia e retornaram à APPA para posterior adoção.

Para avaliar se houve diferença significativa entre os parâmetros clínicos e resultados laboratoriais foi utilizada a análise de variância (ANOVA) de duas vias com múltiplas comparações pelo teste de Tukey para os dados que apresentavam distribuição normal, sendo os dados registrados como média e desvio-padrão, e o teste de Friedman para os dados que apresentavam distribuição não normal, sendo os dados apresentados como mediana e desvio interquartílico. Para avaliação dos escores de estresse utilizou-se o teste Friedman. Para os dados em que foram comparadas apenas duas amostras, foi utilizado o teste $t$ de Student. Aplicou-se um nível de $5 \%$ de significância utilizando-se o programa BioEstat 5.0. 
Tabela 1. Critérios de avaliação do grau de estresse.

\begin{tabular}{clc}
\hline \multicolumn{1}{c}{ CRITÉRIO } & ESCORE \\
\hline \multirow{3}{*}{ PARÂMETRO } & Atento e responde à voz e toques humanos & 0 \\
& Responde timidamente & 1 \\
& Não responde imediatamente & 2 \\
& Não responde ou responde agressivamente & 3 \\
& $</=10 \%$ maior que o valor basal & 0 \\
FC, FR, PA & 11 a $30 \%$ maior que o valor basal & 1 \\
& 31 a $50 \%$ maior que o valor basal & 2 \\
& $>50 \%$ maior que o valor basal & 3 \\
\multirow{2}{*}{$\mathrm{T}^{\circ} \mathrm{C}$} & Normal (37.5-39.0 $\left.{ }^{\circ} \mathrm{C}\right)$ & 0 \\
& Acima de 39 ${ }^{\circ} \mathrm{C}$ & 1 \\
& Vecalização vocalização & 0 \\
& Vocalização presente e controlada & 1 \\
& Vocalização presente não controlada & 2 \\
& Adormecido ou calmo & 0 \\
Agitação & Leve agitação & 1 \\
& Moderada agitação & 2 \\
& Severa agitação & 3 \\
\hline
\end{tabular}

Fonte: adaptado de Beerda et al. (1998) e Hiby et al. (2006).

\section{Resultados e Discussão}

Não houve diferença significativa entre os pesos dos animais ao chegarem ao canil $(12,96 \pm 3,19 \mathrm{~kg})$ e após o M168 $(13,55 \pm 3,29 \mathrm{~kg})$, demonstrando que o confinamento não influenciou no peso corpóreo.

Ao se analisar os resultados laboratoriais de cortisol sérico demonstrados na Tabela 2, observou-se que a concentração média de cortisol detectada no M0 excedeu os valores fisiológicos citados para a espécie canina, compreendidos entre 0,25 e $2,3 \mu \mathrm{g} / \mathrm{dL}$ (Russell et al., 2007). Esse fato pode ser explicado pelas condições em que os animais viviam, as quais, por si só, já eram consideradas estressantes. A equipe estava preparada para reduzir o estresse dos animais ao mínimo, porém, o primeiro contato com os pesquisadores e a contenção física podem ter influenciado nos achados de cortisol basal. Além disso, os animais viviam em um ambiente de forma conjunta e tiveram que ser separados e levados para uma sala próxima onde pudesse ser realizada a coleta de sangue. Tal conduta pode ter assustado os animais que nunca haviam saído do abrigo, causando estresse nesse momento.

Em todos os momentos após o M0, nos quais os animais foram avaliados, coletou-se sangue imediatamente após o contato com o animal, não havendo influência dos picos hormonais decorrentes da manipulação, considerando que o pico de cortisol em cães tende a ocorrer de 15 a 30 minutos após o estímulo doloroso ou estressante (Inoue et al., 2006).

Nos demais momentos houve diminuição gradativa dos níveis de glicocorticoides, devido, provavelmente, à diminuição do estresse pela adaptação ao local e aos pesquisadores, ou a uma habituação à técnica de avaliação dos parâmetros fisiológicos. Esses níveis mantiveram-se dentro dos valores fisiológicos para a espécie canina já a partir do primeiro dia no novo ambiente o que pode ser explicado pelo fato de os animais serem provenientes da associação de cães abandonados onde há constante contato com diferentes pessoas que visitam esses abrigos concordando com Hennessy (2013) que afirma que a interação humana constante reduz a resposta do cortisol a distúrbio em momento posterior. Além disso, experiência com estressores crônicos no abrigo de origem, tais como, competição por alimento e espaço, agressividade por parte dos outros animais e convivência com animais doentes, podem ter reduzido a magnitude da resposta do cortisol ao confinamento no novo ambiente (Hennessy et al., 2001).

Houve diferença significativa nos níveis de cortisol sérico, em relação ao momento basal, apenas a partir do M144, ou seja, seis dias após a chegada dos animais ao canil, propondo-se que a partir desse momento houve maior adaptação dos animais ao local e aos pesquisadores. 
A máxima concentração de glicemia (Tabela 2) observada no momento basal pode ser explicada pelos picos hormonais de cortisol anteriormente discutidos, uma vez que a elevação do cortisol determina aumento da glicogênese hepática (Romero e Butller, 2007).

Tabela 2. Cortisol sérico (mediana \pm desvio interquartílico) e glicemia (média \pm desvio padrão) em cadelas submetidas a um período de adaptação de sete dias em canis experimentais.

\begin{tabular}{lcccccccc}
\hline & M0 & M24 & M48 & M72 & M96 & M120 & M144 & M168 \\
\hline Cortisol & $2,78 \pm$ & $2,14 \pm$ & $1,83 \pm$ & $1,63 \pm$ & $1,32 \pm$ & $1,03 \pm$ & $0,60 \pm$ & $0,55 \pm$ \\
$(\mu \mathrm{g} / \mathrm{dL})$ & 2,7 & 1,36 & 1,28 & 1,03 & 1,13 & 0,71 & $0,67^{*}$ & $0,55^{*}$ \\
Glicose & $101,9 \pm$ & $84,4 \pm$ & $81,3 \pm$ & $80,1 \pm$ & $81,6 \pm$ & $78,7 \pm$ & $79,4 \pm$ & $81,6 \pm$ \\
$(\mathrm{mg} / \mathrm{dL})$ & 11,4 & $4,9^{*}$ & $9,0^{*}$ & $7,5^{*}$ & $5,6^{*}$ & $6,9^{*}$ & $5,7^{*}$ & $6,7 *$ \\
\hline
\end{tabular}

Valor superior: média ou mediana.

Valor inferior: desvio padrão ou interquartílico.

* - diferente do M0 segundo o teste de Friedman ou ANOVA $(\mathrm{p}<0,05)$.

A manutenção da frequência cardíaca dentro dos parâmetros fisiológicos para cães (Massone, 2011), e a ausência de diferença significativa entre os momentos avaliados ( $p>0,05)$ (Tabela 3), discordam de Bergamasco et al. (2010) que afirmam que o aumento no nível de estresse é refletido pelo aumento na frequência cardíaca. Nesse caso, esse parâmetro fisiológico não pôde ser considerado um bom indicador de estresse.

Tabela 3. Mediana \pm desvio interquartílico da frequência cardíaca (FC), frequência respiratória $(f)$, temperatura retal (TR) e pressões arteriais sistólica (PAS), média (PAM) e diastólica (PAD) em cadelas submetidas a um período de adaptação de sete dias em canis experimentais.

\begin{tabular}{ccccccc}
\hline Momentos & $\begin{array}{c}\text { FC } \\
(\mathrm{bpm})\end{array}$ & $\begin{array}{c}f \\
(\mathrm{mpm})\end{array}$ & $\begin{array}{c}\text { TR } \\
\left({ }^{\circ} \mathrm{C}\right)\end{array}$ & $\begin{array}{c}\text { PAS } \\
(\mathrm{mmHg})\end{array}$ & $\begin{array}{c}\text { PAM } \\
(\mathrm{mmHg})\end{array}$ & $\begin{array}{c}\text { PAD } \\
(\mathrm{mmHg})\end{array}$ \\
\hline M0 & $89 \pm 27$ & $40 \pm 35,5$ & $38,9 \pm 0,47$ & $135 \pm 10$ & $107 \pm 13,75$ & $90 \pm 10$ \\
M12 & $106 \pm 36,5$ & $28 \pm 10$ & $38 \pm 0,37$ & $130 \pm 7,5$ & $97 \pm 10$ & $80 \pm 17,5$ \\
M24 & $118 \pm 27$ & $62 \pm 25$ & $38,7 \pm 0,57$ & $135 \pm 17,5$ & $98,5 \pm 6$ & $80 \pm 0$ \\
M36 & $109 \pm 22$ & $37 \pm 21$ & $37,95 \pm 0,35^{*}$ & $120 \pm 10$ & $98,5 \pm 16,25$ & $80 \pm 10$ \\
M48 & $113 \pm 23$ & $58 \pm 30$ & $38,7 \pm 0,45$ & $135 \pm 20$ & $93 \pm 13,75$ & $80 \pm 10$ \\
M60 & $102 \pm 16,5$ & $22 \pm 7$ & $38 \pm 0,52^{*}$ & $120 \pm 17,5$ & $93 \pm 5,25$ & $80 \pm 15$ \\
M72 & $114 \pm 19$ & $56 \pm 35$ & $38,45 \pm 0,25$ & $125 \pm 10$ & $93 \pm 8,5$ & $80 \pm 7,5$ \\
M84 & $122 \pm 44$ & $27 \pm 12$ & $38,10 \pm 0,37^{*}$ & $125 \pm 10$ & $93,5 \pm 13$ & $80 \pm 0$ \\
M96 & $110 \pm 41$ & $58 \pm 43$ & $38,6 \pm 0,07$ & $130 \pm 20$ & $91,5 \pm 9,25$ & $80 \pm 17,5$ \\
M108 & $94 \pm 26$ & $28 \pm 10$ & $38,2 \pm 0,52$ & $130 \pm 10$ & $95 \pm 16$ & $80 \pm 15$ \\
M120 & $116 \pm 24$ & $66 \pm 70$ & $38,7 \pm 0,57$ & $130 \pm 20$ & $88 \pm 10$ & $80 \pm 17,5$ \\
M132 & $120 \pm 30$ & $38 \pm 21$ & $38,05 \pm 0,27$ & $120 \pm 10$ & $103,5 \pm 16^{*}$ & $70 \pm 10^{*}$ \\
M144 & $98 \pm 24$ & $76 \pm 32$ & $38,75 \pm 0,37$ & $135 \pm 27,5$ & $91,5 \pm 12$ & $85 \pm 10$ \\
M156 & $110 \pm 12$ & $24 \pm 7$ & $38,2 \pm 0,77$ & $120 \pm 17,5$ & $86,8 \pm 8,1^{*}$ & $80 \pm 10$ \\
M168 & $110 \pm 30$ & $50 \pm 23,5$ & $38,5 \pm 0,35$ & $125 \pm 10$ & $95 \pm 6,5$ & $80 \pm 7,5$ \\
\hline
\end{tabular}

* - diferente do M0 segundo o teste de Friedman $(\mathrm{p}<0,05)$.

Não ocorreu diferença estatística na frequência respiratória entre os momentos, apesar de Brun et al. (2004) afirmarem que esse parâmetro é útil para avaliação do estresse.

Apenas os momentos M36, M60 e M84 apresentaram temperaturas retais menores estatisticamente que os valores basais, porém sem relevância clínica, uma vez que estas se mostraram dentro dos padrões de normalidade para a espécie em todos os momentos (Feitosa, 2008).

A PAS não variou estatisticamente entre os momentos, mostrando-se discretamente elevada (Cabral et al., 2010) em todos os momentos com exceção do M36, M60, M132 e M156, em que os valores encontraram-se normais para a espécie canina. As PAM e PAD variaram apenas em um 
momento em relação ao momento basal, sendo que a PAD mostrou-se discretamente elevada (Cabral et al., 2010) apenas no M0, devido provavelmente ao estresse sofrido pelos animais durante a aferição dos parâmetros e contenção física. Vale salientar que, apesar de não conhecerem o aparelho de mensuração, os animais não reagiram de forma estressante durante as avaliações de pressão.

Em relação à avaliação clínica do estresse, no ambiente em que os animais viviam, estes se apresentaram calmos e responsivos aos manipuladores, apesar do nível sérico de cortisol estar alto. Já durante o período experimental em todos os momentos o estresse foi classificado como discreto (escores <6). Porém, não se pode dizer que os animais não estavam estressados uma vez que a ativação do eixo hipotálamo-pituitária-adrenal nem sempre se reflete em comportamento, sendo possível que índices hormonais estejam elevados, enquanto os animais aparentam calma (Helmreich et al., 2012). Em relação às alterações funcionais e comportamentais, duas cadelas (20\%) apresentaram-se hiperativas com presença de vocalização em todos os momentos avaliados; dois animais (20\%) mostraram-se agressivos e com medo até 85 horas após a chegada ao canil; dois animais $(20 \%)$ apresentaram diarreia nas primeiras 25 horas; um animal (10\%) estava anoréxico e com apatia até 37 horas após a chegada ao canil; e dois animais (20\%) apresentaram baixa ingestão de ração em todos os momentos. A presença de hiperatividade, agressividade e vocalização acentuada podem ter ocorrido devido à presença de ansiedade, concordando com Tuber et al. (1996) que, estudando o comportamento de cães mantidos em isolamento social em canis individuais por um período de oito semanas, observaram alterações comportamentais, com sinais de irritabilidade e ansiedade, expressos mediante vocalização e atividade locomotora intensa. Porém, essas alterações podem ser decorrentes do comportamento intrínseco do animal.

\section{Conclusão}

O nível de estresse em cadelas de abrigo submetidas a um período de adaptação de sete dias em ambiente diferente foi considerado discreto devido, provavelmente, à presença de fatores mais estressantes no ambiente de origem. Com base nos resultados dos níveis de cortisol sugere-se que seis dias são suficientes para esses animais se adaptarem a um novo ambiente. Mais estudos, com um número maior de animais, são necessários para determinar a validade das mensurações realizadas nesse experimento como indicadores de estresse.

\section{Conflito de interesse}

Os autores declaram não existir conflito de interesse.

\section{Comitê de Ética}

Este estudo foi aprovado pela Comissão de Ética no Uso de Animais da Universidade Federal de Campina Grande, Campus de Patos, Paraíba (protocolo $n^{\circ}$ 42/2012).

\section{Referências}

Beerda, B.; Schilder, M.B.H.; Hoof, J.A.R.A.M.; deVries, H.W.; Mol, J.A. Behavioural, saliva cortisol and heart rate responses to different types of stimuli in dogs. Applied Animal Behavior Science, 58(3): 365-81, 1998.

Beerda, B.; Schilder, M.B.; Bernadina, W.; De Vries, H.W.; Vanhooff, J.A.; Mol, J.A. Chronic stress in dogs subjected to social and spatial restriction: 2. Hormonal and immunological responses. Physiology \& Behavior, 66(2): 243254, 1999.

Bergamasco, L.; Osella, M.C.; Savarino, P.; Larosa, G.; Ozella, L.; Manassero, M.; Badino, P.; Odorec, R.; Barbero, R.; Giovann, Re. Heart rate variability and saliva cortisol assessment in shelter dog: Human-animal interaction effects. Applied Animal Behaviour Science, 125(1): 56-68, 2010.

Brun, M.V.; Pippi, N.L.; Beck, C.A.C.; Contensini, E.A.; Pereira, R.A.; Stedile, R.; Bonfada, A.T.; Columé, L.M.; Gomes, K.; Júnior, A.R.P.V.; Silva, T.F. Colopexiaincisional por celiotomia ou transparietal auxiliada por laparoscopia em cães. Ciência Rural, 34(3): 829-837, 2004.

Budziak, C.; Anater, A.; Villanova Junior, J.A.; Mangrich, R.M.V.; Catapan, D.C.; Pimpão, C.T. Perfis clínico e laboratorial de cães de abrigo submetidos a esterilização. Revista Brasileira de Ciência Veterinária, 23(3): 157162, 2016.

Cabral, R.R.; Ciasca, B.D.; Oliveira, V.M.C.; Vazcurado, A.P.; Larsson, M.H.M.A. Valores da pressão arterial em cães pelos métodos oscilométrico e Doppler vascular. Arquivo Brasileiro de Medicina Veterinária e Zootecnia, 62(1): 64-71, 2010.

Cassu, R.N.; Andreazi, C.D.; Pereira, L. Efeito da Matricaria chamomilla $\mathrm{CH} 12$ na resposta de 
estresse em cães. Colloquium Agrariae, 7(2): 01-07, 2011.

Feitosa, F.L.F. Exame Físico Geral ou de Rotina. In:___ Semiologia veterinária: a arte do diagnóstico. $2^{\mathrm{a}}$ ed. São Paulo: Roca, 2008. p.77-102.

Haverbeke, A.; Diederich, C.; Depiereux, E.; Giffroy, J.M. Cortisol and behavioral responses of working dogs to environmental challenges. Physiology \& Behavior, 93(1): 59-67, 2008.

Helmreich, D.L.; Tylee, D.; Christianson, J.P.; Kubala, K.H.; Govindarajan, S.T.; O’Neill, W.E.; Becoats, K.; Watkins, L.; Maier, S.F. Active behavioral coping alters the behavioral but not the endocrine response to stress. Psychoneuroendocrinology, 37(12): 19411948, 2012.

Hennessy, M.B.; Davis, H.N.; Williams, M.T.; Mellott, C.; Douglas, C.W. Plasma cortisol levels of dogs at a county animal shelter. Physiology \& Behavior, 62(3): 485-490, 1997. Hennessy, M.B.; Voith, V.L.; Mazzei, S.J.; Buttram, J.; Miller, D.D.; Linden, F. Behavior and cortisol levels of dogs in a public animal shelter, and an exploration of the ability of these measures to predict problem behavior after adoption. Applied Animal Behavior Science, 73(3): 217-233, 2001.

Hennessy, M.B. Using hypothalamic-pituitaryadrenal measures for assessing and reducing the stress of dogs in shelters: A review. Applied Animal Behaviour Science, 149(1): 1- 12, 2013.

Hiby, E.F.; Rooney, N.J.; Bradshaw, J.W.S. Behavioural and physiological responses of dogs entering re-homing kennels. Physiology \& Behavior, 89(3): 385-391, 2006.

Inoue, T.; Ko, J.C.; Mandsager, R.E.; Payton, M.E.; Galloway, D.S.; Lange, D.N. Efficacy and safety of preoperative etodolac and butorphanol administration in dogs undergoing ovariohysterectomy. Journal of the American Animal Hospital Association, 42(3): 178-188, 2006.

Massone, F. Anestesiologia veterinária farmacologia e técnicas, texto e atlas. $6^{\mathrm{a}}$ ed. Rio de Janeiro: Guanabara Koogan, 2011. 428p.
Mcewen, B.S.; Gianaros, P.J. Central role of the brain in stress and adaptation: Links to socioeconomic status, health and disease. Annals of the New York Academy of Sciences, 1186: 190-222, 2010.

Mormède P.; Andanson, S.; Aupérin, B.; Beerda, B.; Guémené, D.; Malmkvist, J.; Manteca, X.; Manteuffel, G.; Prunet, P.; VanReenen, C.G.; Richard, S.; Veisser, I. Exploration of hypothalamic-pituitary-adrenal function as a tool to evaluate animal welfare. Physiology \& Behavior, 92(3): 317-339, 2007.

Part, C.E.; Kiddie, J.L.; Hayes, W.A.; Mills, D.S.; Neville, R.F.; Morton, D.B.; Collins, L.M. Physiological, physical and behavioural changes in dogs (Canis familiaris) when kennelled: Testing the validity of stress parameters. Physiology \& Behavior, 133: 260271, 2014.

Romero, L.M.; Butler, L.K. Endocrinology of stress. International Journal of Comparative Psychology, 20(2): 89-95, 2007.

Rooney, N.J.; Gaines, S.A.; Bradshaw, J.W.S. Behavioural and glucocorticoid responses of dogs (Canis familiaris) to kennelling: investigating mitigation of stress by prior habituation. Physiology \& Behavior, 92(5): 847-54, 2007.

Russell, N.J.; Foster, S.; Clark, P.; Robertson, I.D.; Lewis, D.; Irwin, P.J. Comparison of radioimmunoassay and chemiluminescent assay methods to estimate canine blood cortisol concentrations. Australian Veterinary Journal, 85(12): 487-494, 2007.

Tuber, D.S.; Sanders, S.; Hennessy, M.B.; Miller, J.A. Behavioral and glucocorticoid. Responses of adult domestic dogs (Canis familiaris) to companionship and social separation. Journal of Comparative Psychology, 110(1): 103-108, 1996.

Yamamoto, K.C.M.; Silva, E.Y.T.; Costa, K.N.; Souza, M.S.; Silva, M.L.M.; Albuquerque, V.B.; Pinheiro, D.M.; Bernabé, D.G.; Oliva, V.N.L.S. Avaliação fisiológica e comportamental de cães utilizados em terapia assistida por animais (TAA). Arquivo Brasileiro de Medicina Veterinária e Zootecnia, 64(3): 568-576, 2012. 